\title{
The meat-scrap hypothesis: small quantities of meat may promote cooperative hunting in wild chimpanzees (Pan troglodytes)
}

\author{
Claudio Tennie • Ian C. Gilby $•$ Roger Mundry
}

Received: 18 February 2008 /Revised: 11 September 2008 / Accepted: 19 September 2008 / Published online: 14 October 2008

(C) The Author(s) 2008. This article is published with open access at Springerlink.com

\begin{abstract}
A common explanation for hunting in groups is that doing so yields a greater per capita caloric benefit than hunting solitarily. This is logical for social carnivores, which rely exclusively on meat for energy, but arguably not for omnivores, which obtain calories from either plant or animal matter. The common chimpanzee, Pan troglodytes, is one of the few true omnivores that regularly hunts in groups. Studies to date have yielded conflicting data regarding the payoffs of group hunting in chimpanzees. Here, we interpret chimpanzee hunting patterns using a new approach. In contrast to the classical assumption that hunting with others maximizes per capita caloric intake, we propose that group hunting is favored because it maximizes an individual's likelihood of obtaining important micronutrients that may be found in small quantities of meat. We describe a mathematical model demonstrating that group hunting may evolve when individuals can obtain micronutrients more frequently by hunting in groups than by hunting solitarily, provided that group size is below a certain threshold. Twenty five years of data from Gombe National Park, Tanzania are consistent with this prediction.
\end{abstract}

\footnotetext{
Communicated By D. Watts

C. Tennie $\cdot$ R. Mundry

Max Planck Institute for Evolutionary Anthropology,

Leipzig, Germany

I. C. Gilby

Department of Anthropology, Harvard University,

Cambridge, MA, USA

C. Tennie $(\bowtie)$

MPI EVAN,

Deutscher Platz 6,

04103 Leipzig, Germany

e-mail: tennie@eva.mpg.de
}

We propose that our 'meat-scrap' hypothesis is a unifying approach that may explain group hunting by chimpanzees and other social omnivores.

Keywords Chimpanzees (Pan troglodytes) · Hunting · Cooperation · Diet · Micronutrients · Macronutrients

\section{Introduction}

Why hunt in groups?

Group hunting presents an apparent paradox. It would seem costly for an individual to incur the costs associated with hunting when it could exploit the actions of others. Individuals should therefore refrain from participating. Nevertheless, group hunting is commonly observed (e.g., African lions, Panthera leo (Scheel and Packer 1991); bottlenose dolphins, Tursiops spp. (Gazda et al. 2005); African wild dogs, Crocuta crocuta (Creel and Creel 1995, 2002)). One possible explanation for its widespread occurrence is that group hunting is 'cooperative', i.e., that an individual's net payoff is higher when hunting with others than when hunting solitarily (Packer and Ruttan 1988; Mesterton-Gibbons and Dugatkin 1992; Clements and Stephens 1995). While there has been debate over exactly how to measure group hunting payoffs (e.g., whether to consider reduced costs as well as increased benefits (Creel 1997; Packer and Caro 1997; Creel and Creel 2002)), most studies use calories to assess whether animals hunt cooperatively. This is logical for social carnivores, which obtain almost all of their energy from meat. Indeed, many species experience an increase in meat per capita and/or net caloric gain as hunting group size increases (meta-analysis; Creel and Creel 2002). 
However, the benefits of group hunting are likely to be different for omnivores, which can choose to obtain calories from either plant or animal matter. The common chimpanzee, Pan troglodytes, is one of the few true omnivores that regularly hunts in groups, thus providing an important case for comparison with social carnivores. (Recently, bonobos have been found to hunt other primates, and there is also some evidence to suggest that they did so in a group (Surbeck and Hohmann 2008)). Chimpanzees prey most frequently upon red colobus monkeys (Procolobus spp.) at all sites where the two species are present (Uehara 1997; Boesch and Boesch-Achermann 2000; Mitani and Watts 2001). To date, the precise nutritional significance of meat for chimpanzees is unknown. Nevertheless, meat comprises a very small proportion of chimpanzee diet. At Gombe National Park, Tanzania, chimpanzees spend less than 5\% of their feeding time consuming meat (Goodall 1986; McGrew 1992). This yields an estimated 55g/day for males and $7 \mathrm{~g}$ /day for females (Boesch and Boesch-Achermann 2000). At Taï National Park, Côte d'Ivoire, males and females eat an average of 180 and $25 \mathrm{~g} /$ day, respectively. However, average values may be somewhat misleading, as there is considerable individual and seasonal variation in meat consumption at both sites. Additionally, these values are difficult to interpret, as the daily energy requirements of wild chimpanzees are likely to vary across sites, and for the most part, are unknown.

Chimpanzees exhibit fission-fusion grouping in which members of the same community form subgroups ('groups' or 'parties' hereafter) of changing size and composition (Nishida 1968; Wrangham and Smuts 1980; Goodall 1986). Hunts are more likely to occur if many adult male chimpanzees are present in a group that encounters red colobus monkeys, a pattern which appears to be ubiquitous across sites (Mahale Mountains National Park, Tanzania - Hosaka et al. 2001; Kibale National Park, Uganda (Ngogo) - Mitani and Watts 2001: (Kanyawara)Gilby and Wrangham 2007; Gombe - Gilby et al. 2006). However, researchers remain divided over the explanation for this phenomenon. At Taï, group hunting appears to be calorically motivated. There, a 'social mechanism' of selective meat sharing apparently ensures that hunters obtain a positive net caloric payoff for participating, while non-hunters rarely receive appreciable quantities of meat (Boesch 1994b; Boesch and Boesch-Achermann 2000). However, at Gombe, individuals do not experience a net caloric energy gain by hunting in groups (Boesch 1994b). Meat per capita decreases with male chimpanzee hunting party size at Gombe (Gilby et al. 2006), and is not affected by party size at Ngogo (Mitani and Watts 2001).

It might be argued that hunting in groups will be favored as long as the energy an individual obtains exceeds the energy expended to acquire it, even if the magnitude of the benefit decreases as group size increases. However, if chimpanzees are motivated primarily by calories, then one would expect an increase in hunting frequency when calories from alternative sources (i.e., ripe fruit) are scarce. In contrast to this prediction, several studies have shown the opposite pattern. Hunts are more likely to occur when diet quality is high at Kanyawara (Gilby and Wrangham 2007) and Gombe (Gilby et al. 2006), and when ripe fruit is plentiful at Ngogo (Mitani and Watts 2001, 2005). At Kanyawara, this effect remains after controlling for party size, which positively correlates with both diet quality and hunting probability (Gilby and Wrangham 2007). These data indicate that obtaining calories is not a primary motivation for hunting at these sites.

The lack of universal support for a caloric explanation for group hunting among chimpanzees has prompted a recent focus on the potential social benefits of acquiring meat. Specifically, researchers have proposed that male chimpanzees seek to obtain meat in order to create opportunities for sharing in return for grooming, coalitionary support (Mitani and Watts 2001), and/or sex (Stanford et al. 1994). While there is still debate over why chimpanzees share meat (for review, see Muller and Mitani 2005), recent studies by Gilby et al. (2006, 2008; Gilby and Wrangham 2007) provide evidence against the hypothesis that chimpanzees base hunting decisions on the social benefits of meat sharing. What then provides the selective pressure for chimpanzee social predation? Here we explore an explanation that does not hinge upon caloric or social benefits.

\section{The meat-scrap hypothesis}

Evidence from many taxa illustrate the importance of acquiring micronutrients, for which animals may invest considerable time and energy. In doing so, they may receive little or no caloric gain. For example, chimpanzees at Gombe spend hours licking areas that are presumably high in micronutrients, including stones from cooking fires (Goodall 1986), a board on which salt had previously been used to preserve a snake skin (Wrangham 1975), and newly poured cement (I. Gilby, personal observation). Geophagy at habitual salt licks provides elephants, Loxodonta africa$n a$, with sodium in areas and seasons when dietary sodium is otherwise low (Holdo et al. 2002). Similarly, sodium acquisition may serve as an important influence on aquatic foraging patterns of moose, Alces alces (Belovsky 1981; Belovsky and Jordan 1981; but see MacCracken et al. 1993), and sodium, calcium, and phosphorous appear to influence the feeding selectivity of lowland gorillas, Gorilla gorilla gorilla (Magliocca and Gautier-Hion 2002). Without the requisite levels of calcium, vitamin $\mathrm{A}$, and phosphorous, animals have been shown to suffer health 
and reproductive costs (Tordoff 2001). Thus, species may experience costly dietary deficiencies even in environments that abound with easily accessible calories (Morrison 1983).

For chimpanzees, meat is a valuable source of micronutrients including vitamin A, vitamin $\mathrm{K}$, calcium, sodium, and potassium (Teleki 1973, 1981; Takahata et al. 1984; Goodall 1986; Boesch 1994a; Stanford 1996; Mitani and Watts 2001; Milton 2003a, b). One critical micronutrient is vitamin $B_{12}$ (Hausfater 1976; Jolly 1972), which is almost completely absent in plants, yet is a necessary component of a balanced primate diet (Altmann 1998; Evans and Kliewar 1964). Thus, primates in captivity suffer from health problems when maintained on diets low in $B_{12}$ (Hamilton and Busse 1978). Other important micronutrients are $\mathrm{B}_{6}$, iron, and zinc, which are also only available in small quantities in plant matter, but are found in very high concentrations in meat (Milton 2003a). Lack of zinc has negative effects on primate health (Altmann 1998). Therefore the consumption of even small quantities of meat can clearly reduce the need for such micronutrients - without having to consume great quantities of plant matter. Consuming a small amount of meat (i.e., meat scraps) may thus considerably decrease dietary bulk, allowing animals to focus instead upon energy-rich plants-independent of their micronutritional value (see Milton 2003a). Note that, while we focus on micronutrients, some macronutrients, such as fat and protein, may also enhance the value of meat scraps by reducing dietary bulk. A strategy of using meat as a source of micronutrients and plants as a source of energy has been proposed as a means by which early humans avoided the evolutionary constraints associated with increased body size, namely reduced mobility and poor diet quality (Milton 1999). Chimpanzees may also use such a strategy, as it seems certain that the micronutrients in meat are important for a balanced diet.

Despite widespread agreement that meat is a valuable source of micronutrients for chimpanzees, studies that attempt to explain group hunting typically measure profitability in calories (Boesch 1994b; Mitani and Watts 2001; Gilby et al. 2006). However, a diet that maximizes caloric intake may result in deficiencies in other nutrients (Altmann 1998). Here we propose that above a certain (low) threshold of meat consumption, the benefit of obtaining meat is not simply associated with the actual amount being consumed. We argue that this may explain why group hunting at most chimpanzee study sites persists, even in the absence of per capita caloric benefits. We describe a mathematical model that builds on the suggestion of Gilby et al. (2008) that if group hunting increases an individual's chances of getting a large enough scrap of meat to satisfy the animal's micronutrient requirements (regardless of the caloric costs of acquiring it), social predation yields a net benefit, even in populations where meat per capita decreases with group size or hunting in groups yields a net energy loss for most participants. This is not to say, however, that the benefits of eating meat are completely independent of the amount consumed, nor that the energetic content of meat is unimportant.

\section{The model}

Assumptions and notation

Our model is based on two simplifying assumptions. First, we assume that all individuals have the same probability of capturing a monkey and second, that all individuals present participate in a hunt and share the meat equally (or are equally likely to obtain a share). Initially, we estimate catch probability (the probability of making a kill) and individual caloric benefit as a function of group size assuming there are no synergistic effects of multiple hunters. This initial model is required as a baseline from which to estimate the magnitude of synergistic effects required for group hunting to be at least as beneficial as hunting solitarily. We build upon this simple model in several steps. We calculate the magnitude of different putative synergistic effects of hunting in groups that would be required to provide individuals with as many calories as when hunting solitarily. These synergistic effects are (a) increased amount of prey captured per hunt, (b) increased individual catch probability, and (c) decreased individual costs per hunt. In the final step, we determine the benefits of group hunting in terms of the probability of obtaining some small amount of meat rather than caloric gain.

Throughout, we denote catch probabilities using ' $P I$ ' for an individual and ' $P G$ ' for the group, both subscripted with the corresponding group size (' $N$ '). We denote individual benefits using ' $B$ ' with a subscript indicating the corresponding group size. In principle, ' $P$ ' and ' $B$ ' should be expressed per unit time, but since such a unit is generally arbitrary and constant throughout our model it can safely be dropped. To keep the equations as simple as possible, we set the benefit of an individual hunting solitarily equal to its catch probability when hunting solitarily (hence $B_{1}=P I_{1}$ ).

Catch probability and caloric benefit in the absence of synergistic effects of group hunting

The probability that an individual chimpanzee obtains a certain amount of prey in a given time span will depend on a variety of factors, including prey size, availability, and the probability of detecting and catching the prey, which can be summarized as ' $P I$ '. Initially, we assume that this probability is the same for each individual and remains the same 
regardless of whether subjects hunt alone or in groups of different sizes (hence, $P I_{1}=P I_{N}$ ). As such, the probability that a group makes a kill (' $P G_{N}$ ') can be calculated as follows: The probability that a subject fails to make a kill is $1-P I_{1}$. The probability that a group (of size $N$ ) fails to make a kill therefore equals $\left(1-P I_{1}\right)^{N}$. Hence, the probability that the group makes a kill is

$P G_{N}=1-\left(1-P I_{1}\right)^{N}$

As can be seen, the probability that the group makes a kill clearly increases with increasing group size (Fig. 1). Note that this equation follows the same logic as the 'additive probability' hunting hypothesis proposed by Gilby et al. (2006, 2008).

Assuming that prey is shared evenly among all chimpanzees in the group, and that neither the total amount of meat obtained per kill nor the individual probabilities of catching prey vary with hunting group size, one can calculate the overall relative benefit per individual (' $B$ ') as a function of group size. This is obtained by dividing the probability that a group makes a kill by the group size, or $B_{N}=P G_{N} / N$ or

$B_{N}=\left(1-\left(1-P I_{1}\right)^{N}\right) / N$

Not surprisingly, this leads to a decreased relative benefit with increased group sizes (Fig. 2). Hence, the increased probability of making a catch when hunting in a group does not compensate for the increased number of subjects sharing the catch. As a consequence, in the absence of synergistic effects, hunting in a group is maladaptive with regard to caloric maximization. In the next step, we will evaluate how large the synergistic effects would have to be in order to render hunting in a group more energetically efficient.

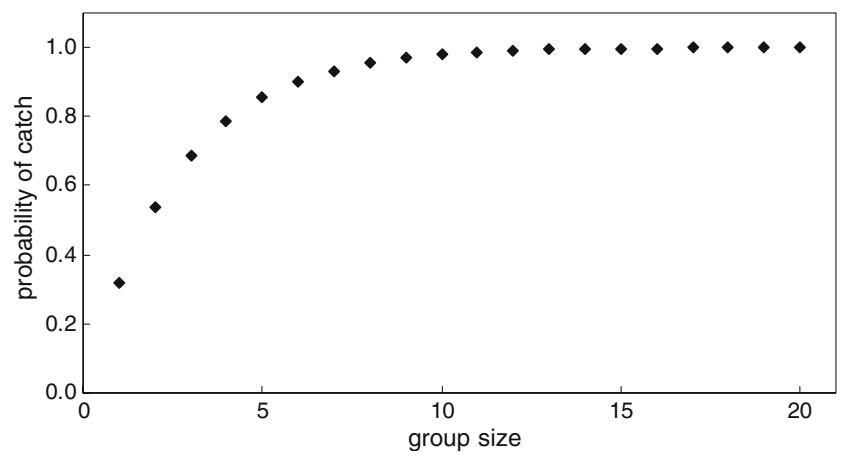

Fig. 1 Overall probability of capturing prey $\left(P G_{N}\right)$ as a nonsynergistic function of group size (Eq. 1). The model is based on the assumptions that each subject has the same probability of capturing prey and that this probability does not vary with group size. Individual catch probability was set to 0.32 , following Gilby et al. (2006)

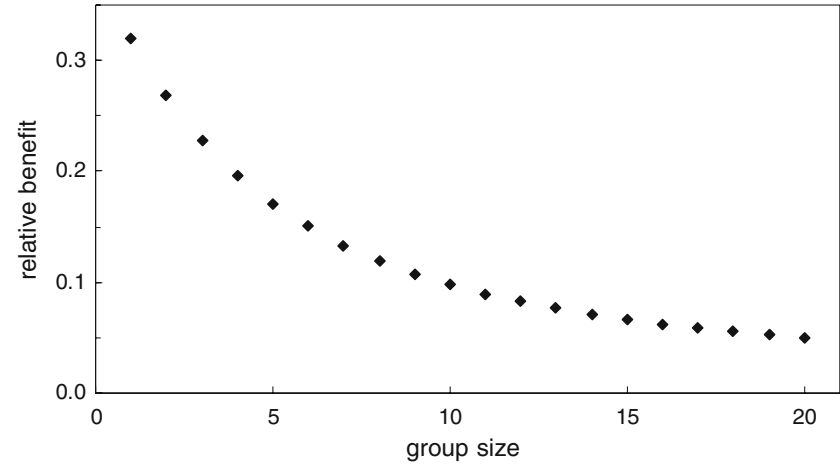

Fig. 2 Individual caloric benefit $\left(B_{N}\right)$ as a function of group size (Eq. 2), assuming the non-synergistic catch probability function of group size depicted in Fig. 1 as well as constant prey size and individual hunting cost. Individual catch probability was set to 0.32 following Gilby et al. (2006). This model indicates that, unless synergistic effects exist, it is not beneficial to hunt in groups. Benefit is displayed in arbitrary units

Magnitude of synergistic effects required for group hunting to be as beneficial as solitary hunting

Because chimpanzees do hunt in groups, we assume that they somehow must compensate for such a decrease. This assumption is justified because, unlike some obligate social carnivores for which group hunting is a by-product of other selective pressures for sociality (e.g., communal cub-rearing in African lions; Packer et al. 1990), the fission-fusion chimpanzee social system provides many opportunities for lone individuals to hunt. Nevertheless, chimpanzees selectively hunt in groups. Thus, hunting with others should be at least as beneficial as solitary hunting. This assumption is supported by empirical data from Gombe and Ngogo, where total kilograms of meat captured increased with hunting party size (Watts and Mitani 2002; Gilby et al. 2006).

Therefore, we incorporate a prey size variable (' $P S_{N}$ ') into the model, making hunting in a group of $N$ subjects as beneficial as solitary hunting, hence, $P S_{N} * B_{N}=B_{1}$, or $P S_{N} *\left(1-\left(1-P I_{1}\right)^{N}\right) / N=B_{1}$. Isolating $P S_{N}$ yields

$P S_{N}=P I_{1} * N /\left(1-\left(1-P I_{1}\right)^{N}\right)$

Since the term $\left(1-P I_{1}\right)^{N}$ asymptotically approaches zero with increasing group size, $P S_{N}$ asymptotically approaches $P I_{1} * N$, a function that is proportional to group size. This means that for hunting in a group to be as beneficial as solitary hunting, there must be a considerable increase in the amount of prey captured per hunt to compensate for sharing. For instance, at Gombe, where the success rate of a lone hunter is $P I_{1}=0.32$ (figure 4 in Gilby et al. 2006), the model predicts that, to compensate for sharing, the amount 
of meat per catch would have to increase by a factor of 3.27 when hunting in a group of ten vs. hunting solitarily. A group of five subjects would have to capture at least 1.87 times more prey than a single individual in order to be beneficial for the individual participants (Fig. 3a). While these values may seem reasonable, the fact that meat per capita significantly decreased with group size at Gombe (Gilby et al. 2006) and was unrelated to group size at Ngogo (Mitani and Watts 2001) suggests that any increase
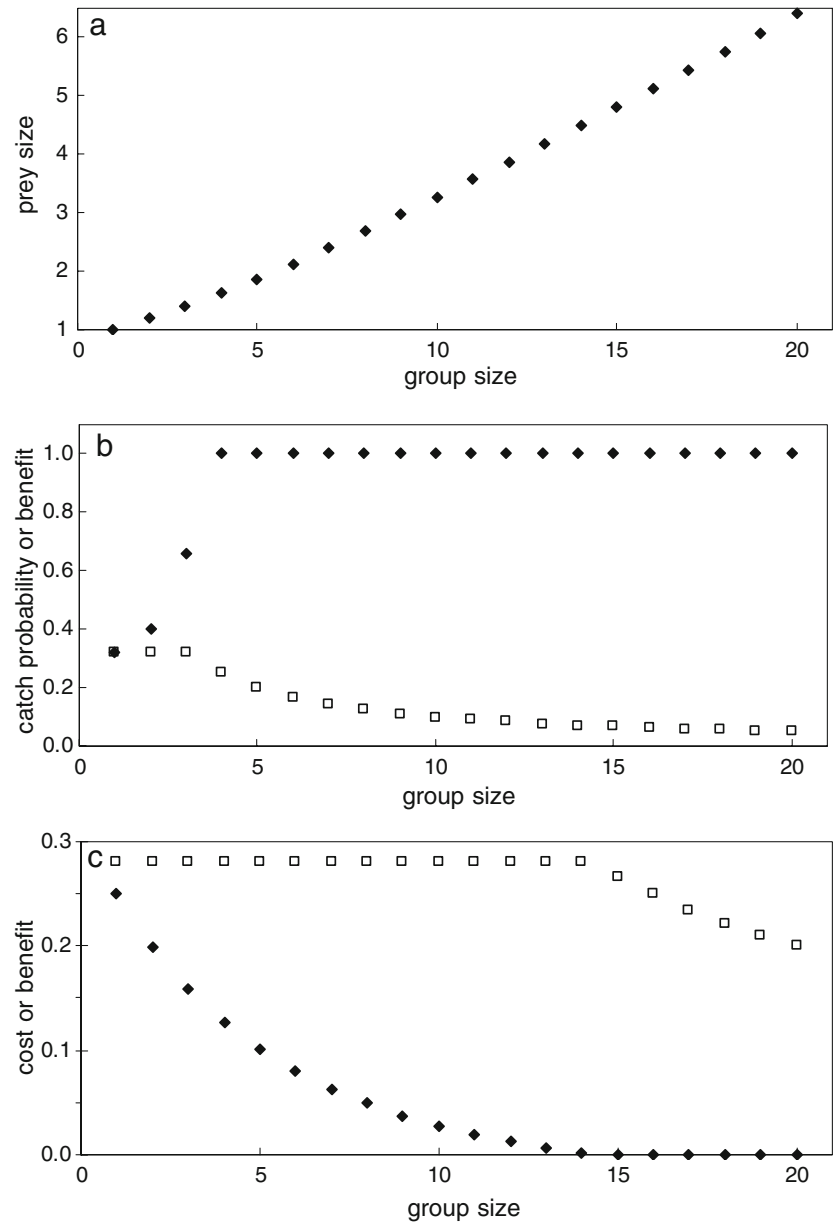

Fig. 3 Compensation (filled diamonds) required in order for hunting in a group to be as beneficial as hunting solitarily in terms of a prey size, $\mathbf{b}$ individual capture probability, and $\mathbf{c}$ individual costs as well as individual benefit (open squares) under the respective model. Prey size, individual catch probability, and individual cost are determined based on the findings shown in Fig. 2 and calculated such that an individual's relative benefit when hunting in a group equals that when hunting solitarily. Note that in (b), increased individual catch probabilities cannot compensate for sharing among four or more subjects, whereas in (c) the reduced costs of hunting in a group cannot compensate for sharing among more than 14 sharing subjects. Individual capture probability was set to 0.32 throughout. Note that synergistic effects have to be rather pronounced in order for hunting in groups to be as beneficial as hunting solitarily. Prey size, costs, and benefits are displayed in arbitrary units in total kilograms of meat obtained by hunting in groups does not or can not fully compensate for the amount lost through sharing.

Our model so far has assumed that the individual catch probability $P I$ is unaffected by party size. However, there may be a synergistic effect of many hunters on individual hunting success. Subjects may actively cooperate, or the simultaneous (yet independent) actions of many individuals may disturb colobus' defenses and increase the probability of a kill. Therefore, we modified the equation such that $P I$ became a function of group size (hence, $P I_{1} \neq$ $\left.P I_{N}\right)$. As before, we assume that for cooperative hunting to evolve, it must be at least as beneficial as solitary hunting, hence, $B_{1}=B_{N}$. Substitution of Eq. (2) yields $P I_{1}=$ $\left(1-\left(1-P I_{N}\right)^{N}\right) / N$, and isolating $P I_{N}$ reveals

$P I_{N}=1-\sqrt[N]{1-P I_{1} * N}$

Since the term $1-P I_{1} * N$ eventually becomes negative as $N$ increases, $P I_{N}$ is not defined for large groups. Hence, we place an upper limit of 1 on $P I_{1} * N$ (which corresponds to placing an upper limit of 1 on $\left.P I_{N}\right)$. As a result, the synergistic effects of group hunting on individual catch probability would need to be considerable for $B_{1}$ to equal $B_{N}$. At Gombe $\left(P I_{1}=0.32\right)$, an individual's catch probability in a group of three $\left(P_{3}\right)$ would have to be increased to at least 0.66 for group hunting to be energetically profitable (Fig. 3b). In a group of four or more, individual catch probabilities must equal one. These values are clearly unrealistic. In fact, at Gombe, the probability that the focal male made a kill did not increase with group size (Gilby et al. 2006), and certainly never approached 1. Even if it did, the individual benefit of hunting in a group of four would be smaller than when hunting in smaller groups, and any further increase in group size would lead to a further reduction in individual benefit due to sharing (Fig. 3b).

An additional mechanism that may render group hunting as beneficial as solitary hunting could be that individuals hunting in groups experience reduced costs. We modelled this by assuming that the individual benefit equals the calories gained per hunt (' $G$ ') minus the caloric cost (' $C$ ') of hunting. Since an unsuccessful hunt also imposes such a cost, the overall caloric benefit per unit time for a subject hunting solitarily is $B_{1}=P I_{1} *$ $\left(G-C_{1}\right)-\left(1-P I_{1}\right) * C_{1}$. Assuming that prey size and hence the overall caloric gain per successful hunt does not vary with the number of hunters and also assuming a constant individual capture probability, the individual caloric benefit for subjects hunting in groups is then $B_{N}=$ $P G_{N} *\left(G / N-C_{N}\right)-\left(1-P G_{N}\right) * C_{N}$. For hunting in a group to be as beneficial as solitary hunting, $B_{N}$ must be 
at least as large as $B_{1}$ or $P G_{N} *\left(G / N-C_{N}\right)-\left(1-P G_{N}\right) *$ $C_{N} \geq P I_{1} *\left(G-C_{1}\right)-\left(1-P I_{1}\right) * C_{1}$. Isolating $C_{N}$ reveals

$C_{N} \leq C_{1}+P G_{N} * G / N-P I_{1}{ }^{*} G$

According to this equation, in which the term $P G_{N} * G / N$ asymptotically approaches zero as group size increases, the cost of hunting in a group may eventually become negative. Hence, we set $C_{N}$ to $C_{1}+P G_{N} * G / N-P I_{1} * G$ if $C_{1}+$ $P G_{N} * G / N-P I_{1} * G \geq 0$ and to zero otherwise. As can be seen, costs would have to decrease very rapidly to compensate for hunting in groups, and above a certain group size, even a cost of zero cannot compensate for the loss due to sharing compared to when hunting solitarily. For instance, with the individual hunting success rate we used here (0.32) and assuming the cost of a lone hunter to be $25 \%$ of the caloric gain per successful hunt (Boesch 1994b) we found that individual costs would need to be decreased by $60 \%$ and $89 \%$ for hunting to be profitable in groups of five and ten, respectively. Hunting in groups of more than 14 individuals would be less beneficial than hunting solitarily. It is worth noting that lower costs for lone hunters would require even steeper cost decreases for group hunting to be as beneficial as solitary hunting. Furthermore, it would lead to a smaller group size threshold above which there is no longer a benefit to hunting in groups.

To summarize, without synergistic effects of multiple hunters, group hunting yields fewer calories per capita than hunting solitarily. Synergistic effects may compensate for the costs of sharing if hunting in groups results in the capture of more prey, increased individual success rates, or reduced individual costs. However, these synergistic effects must be of considerable (and generally unrealistic) magnitude for increased caloric benefits to promote group hunting.

\section{Meat-scrap maximization model}

According to the meat-scrap hypothesis, there are hunting benefits that are not proportional to the amount of meat (i.e., calories) obtained. Instead, an individual's benefit is simply based on the probability of obtaining enough meat to satisfy its micronutrient requirements. This is a nonlinear function of (1) the group's probability of making a catch and (2) the number of individuals which can satisfy their micronutrient requirements from a single catch. A subject's benefit per catch is therefore at a maximum when the number of individuals that share the catch is below a certain group size threshold (i.e., when all individuals can satisfy their micronutrient needs). This function, in turn, must be combined with the group's catch probability to yield the overall individual benefit per unit time. Again, we assume that neither individual capture probability $(P I)$ nor prey amount varies with group size. Also, we assume that prey is shared among a number of group members, allowing all of them to satisfy their meat scrap needs (' $N_{\mathrm{MS}}$ '). Hence, if the group's probability of making a catch $\left(P G_{N}\right)$ times the individual's probability of receiving a sufficient amount $\left(N_{\mathrm{MS}} / N\right)$ is above or equal to $B_{1}$, we set an individual's relative benefit as $B_{\mathrm{N}}=B_{1}$. If $P G_{N}$ is below $B_{1}$, then we set $B_{\mathrm{N}}$ equal to the group's probability of making a catch times the individual's probability of receiving a sufficient amount. Hence, $B_{\mathrm{N}}=B_{1}$ if $P G_{N}$ * $N_{\mathrm{MS}} / N \geq B_{1}$, otherwise $B_{N}=P G_{N} * N_{\mathrm{MS}} / N$. In terms of an individual's probability of capturing prey, these equations become $B_{N}=B_{1}$ if $\left(1-\left(1-P I_{1}\right)^{N}\right) * N_{\mathrm{MS}} / N \geq B_{1}$ and otherwise $B_{N}=\left(1-\left(1-P I_{1}\right)^{N}\right) * N_{\mathrm{MS}} / N$. As group size $(N)$ increases, the term $1-\left(1-P I_{1}\right)^{N}$ will asymptotically approach one. Hence, the group size until which hunting in a group is as beneficial as hunting solitarily approximately equals $N_{\mathrm{MS}} / P I_{1}$. This means that, as long as group size is below this threshold, the relative individual benefit is not related to the number of subjects sharing the catch (Fig. 4, open squares). It also means that when group size is above that threshold the relative individual benefit is a function of the group's probability of making a catch and the probability of a subject acquiring sufficient scraps. Under such a model, it would neither be costly nor beneficial for an individual to hunt in groups as long as group size remains below the aforementioned threshold. As such, there would be no selective pressure favoring the evolution of group hunting. However, this model does not incorporate physiological changes over time.

We can resolve this problem using the logical assumption that acquiring a certain amount of micronutrients does not satisfy the corresponding need forever and thus occasional 'refilling' will be desirable. Hunting in a group can therefore be beneficial because the increased probability of a group making a kill leads to reduced intervals between catches. Hence, we defined $B_{N}=P G_{N} * N_{\mathrm{MS}} / N$ or $B_{N}=\left(1-\left(1-P I_{1}\right)\right)^{N} * N_{\mathrm{MS}} / N$. Under such a scenario, an individual's relative benefit of hunting in a group could rapidly increase with increasing group size until a maximum benefit is reached when group size equals the number of subjects that can obtain sufficient scraps (Fig. 4, filled diamonds). Above that group size threshold, an individual's relative benefit decreases below the maximum but remains greater than the payoff for solitary hunting as long as $B_{N}>P I_{1}$, or, to put it in terms of the individual capture probability, as long as $\left(1-\left(1-P I_{1}\right)^{N}\right) * N_{\mathrm{MS}} / N>P I_{1}$. As before, the group size threshold above which hunting in a group is less beneficial than hunting solitarily approximately equals $N_{\mathrm{MS}} / P I_{1}$. Such a curve is remarkably consistent with empirical data from Kanyawara (figure 2 in Gilby et al. 2008). Note that this model assumes that a subject can 


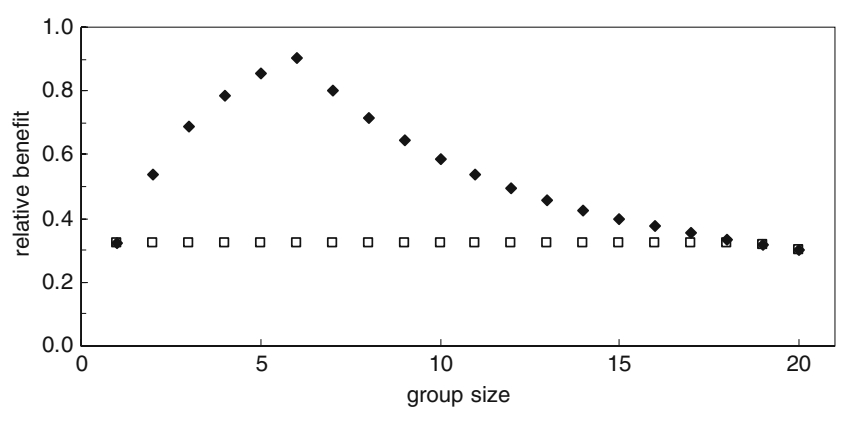

Fig. 4 Relative individual benefit assuming that obtaining a scrap of meat represents a benefit. Open squares show a model based on the assumption that fulfillment of micronutrient needs is at a maximum as long as individual meat-scrap needs are fulfilled at each hunt. Filled diamonds depict a model in which decreased intervals between catches lead to better fulfillment of micronutrient needs. Individual capture probability was set to 0.32 following Gilby et al. (2006) and the number of subjects able to satisfy their meat-scrap needs at a given kill was arbitrarily set to six. Modification of these parameters changes the shape, but not the overall pattern of these results. Benefit is displayed in arbitrary units

make use of a certain amount of micronutrients each time the group makes a kill. If this were not the case, i.e., shorter intervals between catches lead to reduced micronutrient needs, then above a certain interval between catches the number of subjects that can fulfill their micronutrient needs on a single catch would be positively related to the catch probability of the group, i.e., $N_{\mathrm{MSN}} \sim N_{\mathrm{MS} 1} * P G_{N}$. In terms of Fig. 4 (filled diamonds) such a scenario would potentially lead to the maximum occurring at smaller group sizes and/or the decrease in benefit after the maximum being less steep.

Sensitivity of the model with regard to parameter values

The results of the model outlined above largely depend upon two variables, the individual catch probability $\left(P I_{1}\right)$ and the number of subjects that can obtain sufficient scraps $\left(N_{\mathrm{MS}}\right)$ at a kill. However, manipulation of these values influences the particular shape of the curves, but not the general patterns. Using different individual catch probabilities $\left(P I_{1}\right)$, for example, does not alter the patterns we found. An increase in group size generally leads to an increased catch probability, whereby an increase in group size by one individual has a greater effect in small groups, and the group's probability of making a catch invariably approaches one (Fig. 1). Also, the relative benefit to a subject assuming a non-synergistic catch probability invariably decreases with increasing group size (Fig. 2) and, regardless of the individual catch probability, this decrease is more pronounced in smaller groups. Correspondingly, the same holds also for the effects of cooperation (increased success or decreased cost) required for hunting in a group to be as beneficial as solitary hunting (Fig. 3a-c) which invariably increase with group size. An increase in group size by one individual requires more compensation in smaller groups. Finally, under the meat-scrap model, an individual's relative benefit will invariably peak at the number of subjects that can fulfill their micronutrient needs at a single catch (Fig. 4). The group size at which individual benefit falls below its benefit when hunting solitarily will generally be roughly equal to the number of subjects which can satisfy their meat scrap needs at a single catch $\left(N_{\mathrm{MS}}\right)$ divided by individual catch probability.

\section{Empirical data}

We used 25 years of archived data to test whether hunting patterns at Gombe are consistent with the meat-scrap hypothesis. The Gombe chimpanzees have been systematically studied since 1974. Each day, a team of observers follows an adult chimpanzee (the 'focal', hereafter) from night-nest to night-nest, systematically recording its feeding, ranging, and social behavior, as well as conspicuous group-level events, such as hunting (see Gilby et al. 2006 for a full description of data collection and methods). Gilby et al. (2006) extracted all encounters with red colobus monkeys from data collected during these follows. We carefully examined the data from 751 hunts that were recorded between 1976 and 2001 (originally reported by Gilby et al. (2006)). "Hunting" was defined as, "climbing in pursuit of one or more monkeys". In order to reduce (but not entirely eliminate) the possibility of mistakenly classifying a 'hunter' as a 'non-hunter' during the chaos of a hunt, we restricted our analyses to the 411 hunts for which the actions of the majority of the adult males had been recorded. In practice, this meant excluding instances when hunters were not explicitly identified (i.e., we excluded hunts with descriptions such as, "FR and others hunted", but not "FR, FD, WL, and GB hunted"). See Gilby et al. (2006) for discussion of observer reliability. We used SAS version 9.1 (SAS institute, Cary, NC, USA) for all statistical analyses.

Gilby et al. (2006) showed that meat per capita (total kilograms captured / number of adult males present) decreased with adult male party size. However, if meat were only distributed among hunters, then it would still be possible for group hunting to be energetically profitable. Our analysis of the 411 hunts showed that meat per hunter (total kilograms captured / number of hunters) was not significantly correlated with the number of hunters (linear regression, $F_{1,410}=0.73, P=0.39$ ). These results demonstrate that there could have been no caloric incentive for hunting in groups, even if meat was only distributed among hunters. 
At 332 of these hunts, the focal individual was an adult male. We used long-term feeding records to test whether this male was more likely to eat meat (in any amount) as a function of the number of hunters. Results were consistent with the meat-scrap hypothesis - the odds that the focal male ate meat at a given hunt increased by $18 \%$ with each additional male that hunted (logistic regression, odds ratio $-1.18, \chi_{1}^{2}=5.48, P=0.019$, repeated measure $=$ focal male). Therefore, if small scraps of meat yield sufficient micronutrients, then individuals at Gombe benefit by hunting in groups. In contrast, neither meat per capita (Gilby et al. 2006) nor net calories (Boesch 1994b) increased with hunting party size.

\section{Discussion}

Our model's design suggested that hunting in a group may greatly enhance an individual omnivore's probability of acquiring meat compared to hunting solitarily, but that caloric benefits decreased with increasing group size. Modifying the model to include synergistic effects, whereby the probability of making a kill and/or the amount of meat captured increased with the number of hunters revealed that there could, in principle, be a caloric advantage to hunting in groups. However, the required magnitude of such synergism is generally inconsistent with published data from Gombe (Gilby et al. 2006) and Ngogo (Mitani and Watts 2001). It is therefore unlikely to fully explain the phenomenon of group hunting across all chimpanzee sites. Finally, group hunting could be favored if it reduced individual costs, but again the required reduction is unrealistically large. In addition, synergistic effects in terms of catch probability or reduced costs placed an upper limit on group size beyond which hunting is less beneficial than hunting solitarily. Under the assumptions of the meat scrap hypothesis, however, group hunting can be beneficial below a certain group size threshold and when individuals can obtain micronutrients more frequently by hunting in groups than hunting solitarily. Below, we discuss the implications of these results with regard to empirical data from several sites.

Group hunting by chimpanzees presents a puzzle. The selective pressure for hunting in groups (which is ubiquitous across populations), instead of hunting solitarily, remains unclear. At Taï, the incentive to hunt with others appears to be caloric (Boesch 1994b, Boesch and BoeschAchermann 2000). However, at Gombe, where meat per capita decreases with party size (Gilby et al. 2006), there is no such energetic benefit (Boesch 1994b). At Ngogo, meat per capita was independent of party size (Mitani and Watts 2001). Why then, do chimpanzees at Gombe and Ngogo hunt in groups?
Here and elsewhere (Gilby et al. 2008), we have proposed that if a chimpanzee that hunts with others is more likely to obtain valuable micronutrients (from a small amount of meat) than he would by solitary hunting, then group hunting would be selected for. We argue that this 'meat-scrap' model may explain why chimpanzees at all sites hunt in groups.

Boesch (1994b) proposed that, at Taï, a 'social mechanism', whereby hunters receive more meat than nonhunters, ensures that there is a caloric incentive for hunting with others. Why would this be true at Taï and not Gombe? Boesch (1994b) and Gilby et al. (2006) have argued that a major difference between Taï and Gombe is the ease with which monkeys can be captured. At Taï, the continuous canopy provides many escape routes for red colobus monkeys, and therefore a successful hunt may require complex behavioral coordination (Boesch 1994b, 2002; Boesch and Boesch-Achermann 2000). At Gombe, by contrast, where the habitat is a mosaic of evergreen forest and deciduous woodland with broken canopy (CluttonBrock and Gillett 1979), it appears to be easier to corner prey in trees without escape routes (Boesch 1994b; Gilby et al. 2006), and therefore active collaboration is rare. Instead, group hunts at Gombe are typically simultaneous, independent attempts in which hunters selfishly take advantage of the actions of others. Therefore, there is no selective pressure for preferential sharing among hunters. At Ngogo, hunting probability increases with male chimpanzee party size, yet once again, it does not appear to be profitable in terms of calories. As at Gombe, collaboration is rare among Ngogo males (Mitani and Watts 2001). Collaboration may be unnecessary at Ngogo because large parties simply overwhelm red colobus troops.

At first, the differences among sites may suggest that group hunting at Taï is the result of selective pressure for calorie maximization, whereas at Gombe or Ngogo, hunters maximize the likelihood of receiving meat scraps. However, we suggest that, at all sites, chimpanzees hunt in groups because doing so increases the chances of obtaining valuable micronutrients. At Taï, where active collaboration creates selective pressure to punish cheaters, meat is shared preferentially among hunters. The end result is that hunters at Taï obtain more calories than non-hunters, even though the decision to hunt was not motivated by calories alone. In other words, caloric profitability may be a by-product of the mode of cooperation required to make a kill. There is no compelling evidence to suggest that Taï chimpanzees should be more 'calorie-motivated' than Gombe chimpanzees. The risks of starvation are relatively low; even during periods of ripe fruit scarcity, chimpanzees can always rely upon terrestrial herbaceous vegetation (Wrangham et al. 1991) or other "fallback" foods for energy. 
Empirical data support the wide applicability of the meat-scrap hypothesis. In this study, we have demonstrated that, at Gombe, an individual's probability of obtaining meat increased with the number of hunters. This is consistent with data from Kanyawara (Gilby et al. 2008) and Ngogo (Watts and Mitani 2002). Similarly, for Taï, where the probability of success increases with the number of hunters (Boesch and Boesch-Achermann 2000) who preferentially share with one another, we can conclude that hunting with others increases an individual's chances of obtaining meat.

Another critical prediction of the model matches existing empirical data very well. We demonstrated that an individual's relative benefit of hunting increases rapidly with group size but reaches a maximum when the number of chimpanzees present equals the number of subjects that can obtain sufficient scraps (Fig. 4). This is remarkably consistent with data from Kanyawara (figure 2 in Gilby et al. 2008). However, this pattern may also be due to a greater success rate of scrounging by non-hunters in large parties, as suggested by Gilby et al. (2008). Nevertheless, both explanations indicate that obtaining scraps is an important determinant of group hunting. Of course, this is not to say that meat has no caloric value. Once in possession of meat, it will certainly be in a chimpanzee's best interests to eat as much as possible.

The putative processes of reinforcement that would lead to increases in cooperation as a direct outcome of consuming micronutrients remain unclear. It might be argued that if chimpanzees cannot perceive the effects of obtaining micronutrients, then there is no incentive to seek small amounts of meat. However, the principles of natural selection do not require such reinforcement. If animals that obtain meat have higher reproductive success than those that do not, there will be selective pressure for individuals to behave in a manner which maximizes their probability of obtaining the optimal amount.

Our model rests on the assumption that (a) meat scraps contain concentrated micronutrients which are (b) valuable for chimpanzees in order to optimize their diets and (c) obtaining those micronutrients below a certain threshold would decrease an individual's fitness. We propose (d) that the micronutrient threshold can be satisfied by acquiring relatively small amounts of meat, and that the value of securely procuring these micronutrients outweighs potential energetic costs. We believe we have provided adequate evidence concerning (a) and (b). However, when it comes to fitness (c and d), we face two problems: One is that current chimpanzee behavior may not be fitness maximizing now, but may have been so in the past (Altmann 1998; Crawford 1998). However, given the considerable energetic costs (Boesch 1994b) and risks of injury associated with hunting, it is unlikely that such behavior would persist if there was not a substantial benefit. The other problem is that we are currently unable to provide an accurate estimate of the amount of meat scraps that would satisfy a chimpanzee's micronutritional needs (and furthermore, these values might even differ between field sites, based on dietary differences). When it comes to determining values for required micro- (and sometimes also macro-) nutrients for any species, surprisingly little is known (Altmann 1998). Thus, we are unable to present absolute values for minimal daily requirements for any of the micronutrients that we know exist in large quantities in meat. However, our model does not depend on such absolute values, since at the very least, given the current state of knowledge on dietary deficits, we can infer some minimal relevant micronutritional requirements.

The model itself does not explicitly distinguish between micronutritional and caloric benefits. It is theoretically possible for group hunting to evolve as long as individuals experience a net caloric benefit (even if the magnitude of the benefit decreases with group size). Thus, our requirement that a meat scrap be large enough to satisfy a certain basic micronutritional need could also be applied to calories. However, given the high energetic costs of hunting (Boesch 1994b), the small size of the scraps that hunters often obtain, and the fact that hunting frequency tends to decrease when ripe fruit is scarce, a small amount of meat is more likely to satisfy a micronutrient threshold than one for calories. To support the micronutrient model, it suffices to show that two conditions are met: (1) meat scraps contain some micronutrients that chimpanzees must acquire to stay healthy and (2) chimpanzees could most efficiently acquire these micronutrients through meat scraps. Vitamin $\mathrm{B}_{12}$ and zinc fulfill the first requirement. As for the second, small overall amounts of animal matter suffice to fulfill micronutrient requirements in other primates for which good data on the exact quantities ingested exist (Altmann 1998).

There is no doubt that group hunting among chimpanzees is a complicated phenomenon that is affected by a multitude of factors, including caloric and social benefits. However, before such mechanisms are accepted, studies must provide evidence against more parsimonious alternatives that generate the same patterns. Clearly, a more complete understanding of chimpanzees' macro- and micronutritional requirements and more precise measures of individual meat consumption are required to achieve this goal.

\section{Conclusions}

The meat-scrap hypothesis provides a simple explanation for why there is a general trend for chimpanzees to hunt in groups. It is not mutually exclusive with regard to 
hypotheses that explain why some parties hunt and others do not. Nevertheless, without an incentive to obtain meat, there would be no reason to join the hunt at all (unless hunting costs are reduced to zero). The meat-scrap hypothesis changes the way we assess payoffs and renders group hunts beneficial for chimpanzee hunters at all sites. Our results are broadly applicable to social and solitary omnivores that frequently choose between searching for meat and foraging on readily available plant material. If an animal does not face an immediate calorie deficit, it may choose to hunt in order to satisfy its micronutrient requirements. Further testing of the meat-scrap model requires detailed analysis of micronutrient need, intake, and availability across populations and species.

Acknowledgements We thank Christophe Boesch, Charles Nunn, Yasmin Möbius, Jessica Ganas, Keith Jensen, David Watts, and five anonymous reviewers for comments on the manuscript. This research was supported by the Max Planck Society. ICG was funded by NSF Grant IIS-0431141. We are extremely grateful to Anne Pusey and the Jane Goodall Institute's Center for Primate Studies at the University of Minnesota for facilitating the compilation of the hunting database from the behavioral records from Gombe National Park, and to the Jane Goodall Institute and the entire Gombe Stream Research Centre staff for their tireless efforts in collecting the data.

Open Access This article is distributed under the terms of the Creative Commons Attribution Noncommercial License which permits any noncommercial use, distribution, and reproduction in any medium, provided the original author(s) and source are credited.

\section{References}

Altmann SA (1998) Foraging for survival: yearling baboons in Africa. University of Chicago Press, Chicago

Belovsky GE (1981) A possible population response of moose to sodium availability. J Mammal 62:613-621

Belovsky GE, Jordan PA (1981) Sodium dynamics and adaptations of a moose population. J Mammal 62:631-633

Boesch C (1994a) Chimpanzees-red colobus monkeys: a predatorprey system. Anim Behav 47:1135-1148

Boesch C (1994b) Cooperative hunting in wild chimpanzees. Anim Behav 48:653-667

Boesch C (2002) Cooperative hunting roles among Taï chimpanzees. Hum Nat 13:27-46

Boesch C, Boesch-Achermann H (2000) The chimpanzees of the Taï forest. Behavioural ecology and evolution. Oxford University Press, Oxford

Clements KC, Stephens DW (1995) Testing non-kin cooperation: mutualism and the Prisoner's Dilemma. Anim Behav 50:527-535

Clutton-Brock TH, Gillett JB (1979) A survey of forest composition in the Gombe National Park, Tanzania. Afr J Ecol 17:131-158

Crawford C (1998) Environments and adaptations: then and now. In: Crawford C, Krebs DL (eds) Handbook of evolutionary psychology. Erlbaum, Mahwah, pp 275-302

Creel S (1997) Cooperative hunting and group size: assumptions and currencies. Anim Behav 54:1319-1324
Creel S, Creel NM (1995) Communal hunting and pack size in African wild dogs, Lycaon pictus. Anim Behav 50:13251339

Creel S, Creel NM (2002) The African wild dog: behavior, ecology, and conservation. Princeton University Press, Princeton

Evans HJ, Kliewar M (1964) Vitamin B12 compounds in relation to the requirements of cobalt for higher plants and nitrogen-fixing organisms. Ann NY Acad Sci 112:735-755

Gazda SK, Connor RC, Edgar RK, Cox F (2005) A division of labour with role specialization in group-hunting bottlenose dolphins (Tursiops truncatus) off Cedar Key, Florida. Proc Royal Soc Lond B Bio 272:135-140

Gilby IC, Wrangham RW (2007) Risk-prone hunting by chimpanzees (Pan troglodytes schweinfurthii) increases during periods of high diet quality. Behav Ecol Sociobiol 61:1771-1779

Gilby IC, Eberly LE, Pintea L, Pusey AE (2006) Ecological and social influences on the hunting behaviour of wild chimpanzees (Pan troglodytes schweinfurthii). Anim Behav 72:169-180

Gilby IC, Eberly LE, Wrangham RW (2008) Economic profitability of social predation among wild chimpanzees: individual variation promotes cooperation. Anim Behav 75:351-360

Goodall J (1986) The chimpanzees of Gombe: patterns of behavior. Harvard University Press, Cambridge

Hamilton WJ, Busse CD (1978) Primate carnivory and its significance to human diets. Bioscience 28:761-766

Hausfater G (1976) Predatory behaviour of yellow baboons. Behaviour 56:44-68

Holdo RM, Dudley JP, McDowell LR (2002) Geophagy in the African elephant in relation to availability of dietary sodium. J Mammal $83: 652-664$

Hosaka K, Nishida T, Hamai M, Matsumoto-Oda A, Uehara S (2001) Predation of mammals by the chimpanzees of the Mahale Mountains, Tanzania. In: Galdikas B, Briggs N, Sheeran L, Shapiro G, Goodall J (eds) All apes great and small, vol. I. African Apes. Kluwer, New York, pp 107-130

Jolly A (1972) Nutritional requirements of subhuman primates. Physiol Rev 52:415-467

MacCracken JG, Van Ballenberghe V, Peek JM (1993) Use of aquatic plants by moose: sodium hunter or foraging efficiency. Can J Zool 71:2345-2351

Magliocca F, Gautier-Hion A (2002) Mineral content as a basis for food selection by western lowland gorillas in a forest clearing. Am J Primatol 57:67-77

McGrew WC (1992) Chimpanzee material culture. Cambridge University Press, Cambridge

Mesterton-Gibbons M, Dugatkin LA (1992) Cooperation among unrelated individuals: evolutionary factors. Q Rev Biol 67:267281

Milton K (1999) A hypothesis to explain the role of meat-eating in human evolution. Evol Anthropol 8:11-21

Milton K (2003a) The critical role played by animal source foods in human (Homo) evolution. J Nutr 133:3886-3892

Milton K (2003b) Micronutrient intakes of wild primates: are humans different. Comp Biochem Physiol Part A 136:47-59

Mitani JC, Watts DP (2001) Why do chimpanzees hunt and share meat. Anim Behav 61:915-924

Mitani JC, Watts DP (2005) Seasonality in hunting by nonhuman primates. In: Brockman DK, van Schaik CP (eds) Seasonality in primates: studies of living and extinct human and non-human primates. Cambridge University Press, Cambridge, pp 215-240

Morrison SD (1983) Nutrition and longevity. Nutr Rev 41:133-142

Muller MN, Mitani JC (2005) Conflict and cooperation in wild chimpanzees. Adv Stud Behav 35:275-331

Nishida T (1968) The social group of wild chimpanzees in the Mahali mountains. Primates 9:167-224 
Packer C, Ruttan L (1988) The evolution of cooperative hunting. Am Nat 132:159-198

Packer C, Caro TM (1997) Foraging costs in social carnivores. Anim Behav 54:1317-1318

Packer C, Scheel D, Pusey AE (1990) Why lions form groups: food is not enough. Am Nat 136:1-19

Scheel D, Packer C (1991) Group hunting behavior of lions-a search for cooperation. Anim Behav 41:697-709

Stanford C (1996) The hunting ecology of wild chimpanzees: implications for the evolutionary ecology of Pliocene hominids. Am Anthropol 98:96-113

Stanford CB, Wallis J, Mpongo E, Goodall J (1994) Hunting decisions in wild chimpanzees. Behaviour 131:1-18

Surbeck M, Hohmann G (2008). Primate hunting by bonobos at LuiKotale, Salonga National Park. Current Biology (in press)

Takahata Y, Hasegawa T, Nishida T (1984) Chimpanzee predation in the Mahale Mountains from August 1979 to May 1982. Int J Primatol 5:213-233

Teleki G (1973) The predatory behavior of wild chimpanzees. Bucknell University Press, Lewisburg
Teleki G (1981) The omnivorous diet and eclectic feeding habits of chimpanzees in Gombe National Park, Tanzania. In: Harding RSO, Teleki G (eds) Omnivorous primates. Gathering and hunting in human evolution. Columbia University Press, New York, pp 303-343

Tordoff MG (2001) Calcium: taste, intake, and appetite. Phys Rev 81:1567-1597

Uehara S (1997) Predation on mammals by the chimpanzee (Pan troglodytes). Primates 38:193-214

Watts DP, Mitani JC (2002) Hunting behavior of chimpanzees at Ngogo, Kibale National Park, Uganda. Int J Primatol 23:1-28

Wrangham RW (1975) The behavioural ecology of chimpanzees in Gombe National Park, Tanzania. Ph.D. thesis, Cambridge University

Wrangham RW, Smuts B (1980) Sex differences in the behavioral ecology of chimpanzees in the Gombe National Park, Tanzania. J Reprod Fertil (Suppl) 28:13-31

Wrangham RW, Conklin NL, Chapman CA, Hunt KD (1991) The significance of fibrous foods for Kibale Forest chimpanzees. Philos T Roy Soc 334:171-178 